ARTICLE

Received 17 Jun 2013 | Accepted 2 Dec 2013 | Published 15 Jan $2014 \quad$ DOl: 10.1038/ncomms4039 OPEN

\title{
Integrin CD11b positively regulates TLR4-induced signalling pathways in dendritic cells but not in macrophages
}

Guang Sheng Ling ${ }^{1}$, Jason Bennett ${ }^{2}$, Kevin J. Woollard ${ }^{3}$, Marta Szajna ${ }^{1}$, Liliane Fossati-Jimack ${ }^{1}$, Philip R. Taylor ${ }^{4}$, Diane Scott ${ }^{1}$, Guido Franzoso ${ }^{2}$, H. Terence Cook ${ }^{1} \&$ Marina Botto ${ }^{1}$

Tuned and distinct responses of macrophages and dendritic cells to Toll-like receptor 4 (TLR4) activation induced by lipopolysaccharide (LPS) underpin the balance between innate and adaptive immunity. However, the molecule(s) that confer these cell-type-specific LPSinduced effects remain poorly understood. Here we report that the integrin $\alpha_{M}$ (CD11b) positively regulates LPS-induced signalling pathways selectively in myeloid dendritic cells but not in macrophages. In dendritic cells, which express lower levels of CD14 and TLR4 than macrophages, CD11b promotes MyD88-dependent and MyD88-independent signalling pathways. In particular, in dendritic cells CD11b facilitates LPS-induced TLR4 endocytosis and is required for the subsequent signalling in the endosomes. Consistent with this, CD11b deficiency dampens dendritic cell-mediated TLR4-triggered responses in vivo leading to impaired T-cell activation. Thus, by modulating the trafficking and signalling functions of TLR4 in a cell-type-specific manner CD11b fine tunes the balance between adaptive and innate immune responses initiated by LPS.

\footnotetext{
${ }^{1}$ Centre for Complement and Inflammation Research, Department of Medicine, Imperial College, Hammersmith Campus, Du Cane Road, London W12 ONN, UK. ${ }^{2}$ Centre for Cell Signalling and Inflammation, Department of Medicine, Imperial College, Hammersmith Campus, Du Cane Road, London W12 ONN, UK. ${ }^{3}$ Renal and Vascular Inflammation Section, Division of Immunology and Inflammation, Department of Medicine, Imperial College, Hammersmith Campus, Du Cane Road, London W12 ONN, UK. ${ }^{4}$ Cardiff Institute of Infection and Immunity, Cardiff University School of Medicine, Tenovus Building, Heath Park, Cardiff CF14 4XN, UK. Correspondence and requests for materials should be addressed to M.B. (email: m.botto@imperial.ac.uk).
} 
ipopolysaccharide (LPS) is responsible for many of the pathogenic effects of Gram-negative bacteria and can also induce a protective adaptive immune response by acting as adjuvant. While diverse cell types can respond to LPS, they may mount qualitatively and quantitatively different responses ${ }^{1}$. The molecules and mechanisms dictating the cell type specificity of the LPS-induced effects remain poorly understood.

Dendritic cells (DCs) and macrophages (MФs) arise from common myeloid precursors and share the ability to sample the tissue environment but have distinct effector functions ${ }^{2}$. Both types of cell sense microbes through pattern-recognition receptors, which initiate downstream signalling events ${ }^{3}$. However, as these cells mediate different immune functions, their LPS response must be tuned to reflect their roles.

Toll-like receptors (TLRs) are the best-characterized patternrecognition receptors. TLR4 binds specifically to LPS and triggers two distinct sequential signalling pathways 3,4 . The first pathway (MyD88-dependent) is initiated from the plasma membrane, requires both MyD88 and TIRAP to activate NF- $\mathrm{KB}$ and initiate cytokine production ${ }^{4}$. TLR4 is then internalized into the endosome where a MyD88-independent pathway is triggered. These second signalling events are controlled by the adaptor molecules, TRAM (TRIF-related adaptor molecule) and TRIF (TIR-domain-containing adapter-inducing interferon- $\beta)^{5}$, which activate Interferon (IFN) Regulatory Factor-3 (IRF3) leading to the subsequent production of type I IFNs and CCL5 (RANTES) $^{6,7}$. Although LPS responses depend on a membranespanning complex formed by TLR4/MD-2, several molecules have been shown to act as co-receptors and/or accessory molecules and to regulate both positively and negatively LPS sensing/signalling ${ }^{8}$. One such regulator is CD11b.

CD11b, which pairs with CD18 to form a heterodimeric type 1 transmembrane receptor (CD11b/CD18; $\beta_{2} \alpha_{\mathrm{M}}$ ) known as Mac-1/ complement receptor 3 (CR3), has been suggested to contribute to the LPS signalling cluster ${ }^{8}$. CD11b is highly expressed on several cell types including MФs and DCs and can bind to multiple ligands such as complement activation products (iC3b/ C3b) and LPS $^{9}$. CR3 activation is mediated by conformational changes often referred to as the 'inside-out' and 'outside-in' signalling pathways ${ }^{10}$.

CR3 plays a critical role in regulating inflammation and antimicrobial immunity ${ }^{11}$. In vitro and in vivo observations have also indicated a role for CD11b in TLR-triggered innate immune responses; however, the nature of this cross-talk remains controversial. On one hand, activation of CR3 through the binding of iC3b or fibrinogen delivers an 'outside-in' signal that leads to the downregulation of LPS-induced inflammation ${ }^{11,12}$. On the other hand, certain anti-CD11b antibodies or soluble mediators can act synergistically with LPS $^{13,14}$. Furthermore, a recent study reported that $\mathrm{CD} 11 \mathrm{~b}$ could inhibit TLR signalling even in the absence of exogenous ligands ${ }^{15}$, whereas many other studies have shown that CD11b binds LPS and cooperates with TLR4 to elicit an optimal LPS response ${ }^{16-19}$.

In the present study, we demonstrate that CD11b can serve as a positive regulator of both TLR4-induced signalling pathways only on myeloid-derived DCs; however, it is dispensable in

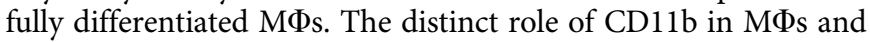
DCs thereby provides an explanation for the apparently conflicting results in the literature and sheds new light on the regulation of the adaptive and innate immune responses triggered by LPS.

\section{Results}

CD11b does not affect TLR4-induced cytokine response in MФs. There is growing interest in understanding how CD11b cross-regulates TLR4 signalling; however, the data in the literature are conflicting and thus the outcome of this cross-regulation remains unresolved ${ }^{14,15,19}$. TLR responses have been often investigated using thioglycolate-elicited peritoneal $\mathrm{M \Phi s}^{15}$. However, as the peritonitis induced by thioglycolate depends on complement activation ${ }^{20}$ and complement-deficient animals, including CD11b-deficient $\left(\right.$ Itgam $\left.^{-1}-\right)$ mice, mount an abnormal cellular response $\mathrm{e}^{20-22}$, we reasoned that resident peritoneal MФs would provide a better controlled model. Consistent with a recent report ${ }^{15}$, we found that thioglycolateelicited Itgam $^{-1-}$ peritoneal MФs produced more interleukin (IL) -6 and tumour necrosis factor alpha (TNF $\alpha$ ) in response to LPS (Fig. 1a). However, these differences were detectable only with thioglycolate-elicited peritoneal MФs, while resident peritoneal MФs or bone marrow-derived MФs (BM-MФs) from Itgam $^{-1-}$ and $\mathrm{W} / \mathrm{T}$ animals secreted equivalent amounts of cytokines (Fig. 1a). Further analysis of the thioglycolate-elicited

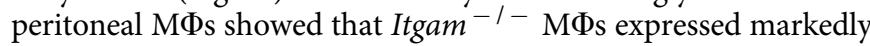
higher levels of major histocompatibility complex (MHC)II than their W/T counterparts, indicating a different state of activation. By contrast, MHCII expression on resident peritoneal MФs and BM-MФs was similar between W/T and Itgam $^{-1-}$ mice (Fig. 1b). Additional analysis of the peritoneal lavage cells from thioglycolate-treated Itgam $^{-1-}$ mice revealed not only that they express higher levels of MHCII, but also that the percentage of $\mathrm{F} 4 / 80^{\mathrm{hi}} \mathrm{CD} 115^{+}$cells was significantly higher (Supplementary Fig. S1). This is consistent with previous reports $^{21,22}$ that $\mathrm{CD} 11 \mathrm{~b}$ deficiency affects the cellular composition of the peritoneal lavage following thioglycolate administration. Collectively, these data demonstrate that CD11b is not involved in the regulation of the LPS-triggered inflammatory response in steady-state $М \Phi$ s.

CD11b promotes MyD88-dependent TLR4 signalling in DCs. We next compared the response of bone marrow-derived DCs (BM-DCs) and BM-MФs over a range of LPS concentrations. We found that Itgam $^{-1}$ - BM-DCs consistently secreted less IL-6 and $\mathrm{TNF} \alpha$, while Itgam $^{-1-}$ BM-MФs did not display any impaired production (Fig. 2a). Consistent with these data, the intensity of TLR4-induced MyD88-dependent phosphorylation of $\mathrm{p} 38$, Erk1/2, JNK and $\mathrm{IkB} \alpha$ was markedly reduced in LPS-stimulated Itgam $^{-1-}$ BM-DCs but not in Itgam $^{-1-}$ BM-MФs compared with the corresponding W/T cells (Fig. $2 \mathrm{~b}$ and Supplementary Fig. S8). Similar cytokine results were observed with both adherent and floating bone marrow-derived myeloid cells differentiated in vitro with granulocyte-macrophage colony stimulating factor (GM-CSF) only (Supplementary Fig. S2b). These cells are variably defined in the literature as MФs or $\mathrm{DCs}^{23-25}$, which may explain some of the conflicting results. Notably, irrespective of the culture conditions applied, Itgam $^{-1-}$ and W/T myeloid cells displayed similar maturation status before LPS stimulation (Supplementary Fig. S2a). Moreover, unstimulated Itgam $^{-/}$and W/T cells expressed similar levels of TLR4 and CD14 (Supplementary Fig. S2a). The presence of GM-CSF in the culture medium is critical for BM-DC differentiation, and thus the different role exhibited by CD11b on BM-DCs and BM-MФs could be the result of GM-CSFmediated cell programming rather than a lineage/differentiationspecific effect, as shown for other molecules ${ }^{26}$. To explore this possibility, we primed Itgam $^{-1-}$ and W/T BM-MФs with GMCSF or GM-CSF plus IL-4 prior to LPS stimulation (Supplementary Fig. S3a). Under these conditions, the cells acquired some DC-like phenotypic features, yet we did not observe any difference in the cytokine production between $\mathrm{W} / \mathrm{T}$ and Itgam $^{-/-}$cells indicating that the CD11b dependency in 

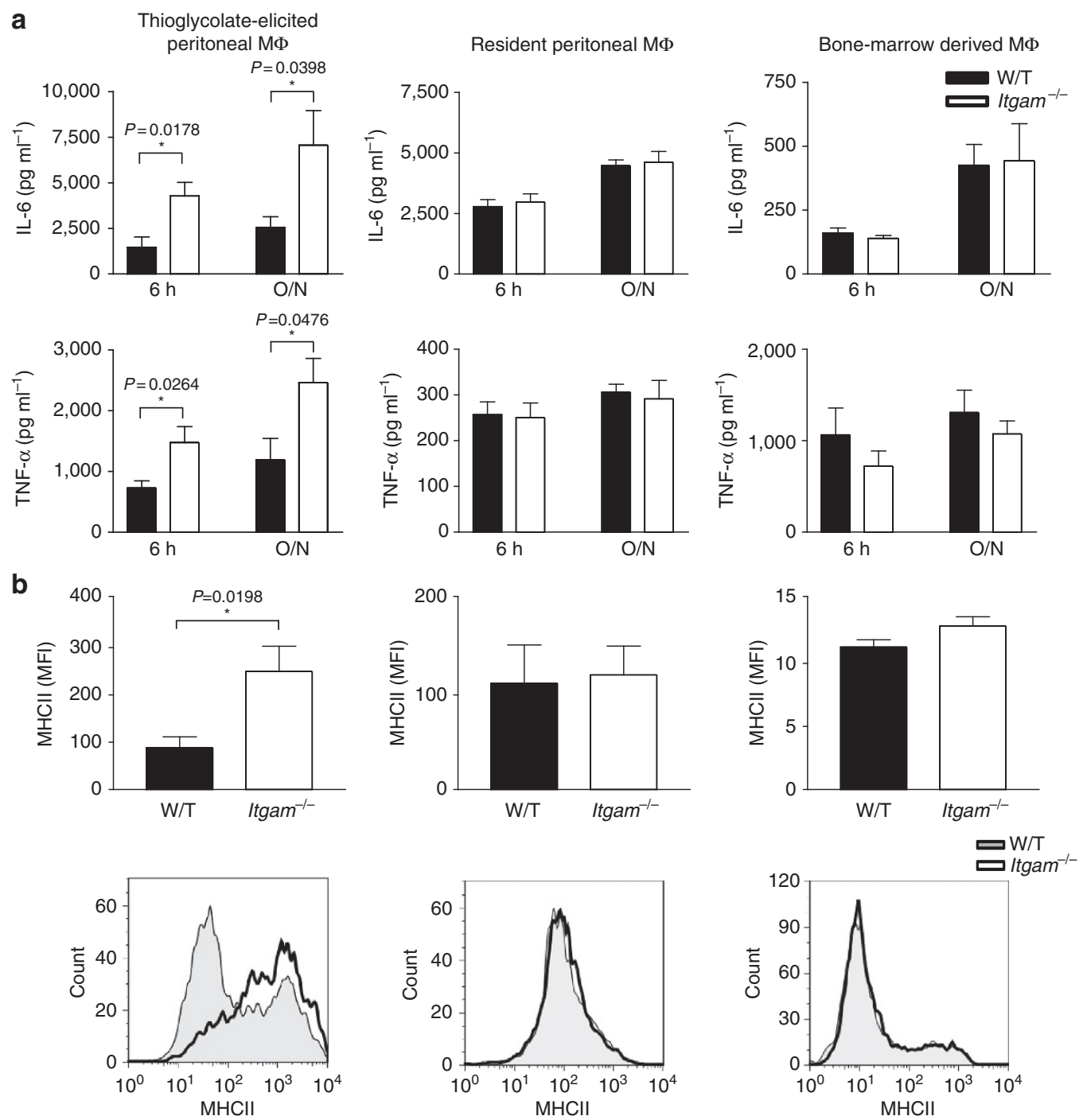

Figure 1 | LPS-induced proinflammatory cytokine production by $\mathbf{M \Phi s}$ is not affected by CD11b deficiency. (a) Thioglycolate-elicited peritoneal M $\Phi$,

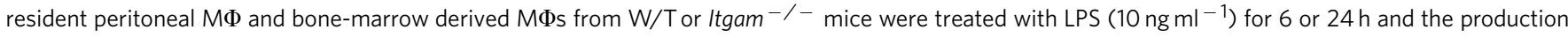
of IL-6 and TNF- $\alpha$ were measured using enzyme-linked immunosorbent assay (ELISA). (b) The surface expression of MHCII on different MФs was examined using flow cytometry prior to the LPS stimulation. Data are expressed as delta MFI (MFI = test-isotype control). Data are represented as mean \pm s.e.m. from two independent experiments with three mice per group. ${ }^{\star} P<0.05$ ( $t$-test).

response to LPS is restricted to BM-DCs (Supplementary Fig. $\mathrm{S} 3 \mathrm{~b}, \mathrm{c})$. As BM-MФs and BM-DCs exhibit different surface levels of TLR4 and CD14 (Supplementary Fig. S2c) and CR3 has been shown to bind LPS, we hypothesized that on cells bearing lower levels of TLR4 and CD14, such as BM-DCs, CD11b is required to promote the formation of the LPS recognition/signalling complex ${ }^{18,19,27}$. We therefore examined CD11b surface distribution after LPS stimulation using multispectral imaging flow cytometry. This analysis showed an increase in the percentage of BM-DCs displaying clustered CD11b after LPS stimulation. In contrast, the surface expression of $\mathrm{CD} 11 \mathrm{~b}$ remained evenly distributed in LPS-treated BM-MФs (Fig. 3). In addition, using an antibody that loses affinity for TLR4 when the receptor interacts with LPS (MTS510) ${ }^{28}$, we found that, upon LPS stimulation, TLR4 staining on Itgam $^{-1-}$ BM-DCs remained largely unchanged, while it was significantly decreased on $\mathrm{W} / \mathrm{T}$ cells (Supplementary Fig. S4a). Collectively, these data demonstrate that CD11b facilitates LPS binding to TLR4 and acts as an additional signalling partner in the LPS receptor signalosome complex on BM-DCs, which express low levels of TLR4 and CD14, but not on
MФs. Thus CD11b contributes to the TLR4 signalling from the plasma membrane in a cell-type-specific manner.

CD11b facilitates TLR4 endocytosis in BM-DCs. Recent work has suggested that TLR4 can be delivered into endosomes, where TRIF-dependent signalling can occur ${ }^{5}$, and that CD14 is essential for this endocytic pathway triggered by LPS $^{3}$. We next explored whether CD11b can contribute to this process. We used the loss of cell surface expression as readout for TLR4 endocytosis and the production of RANTES as a surrogate for TRIF-mediated IRF3 activation $^{3,6,7}$. As shown with the TLR4 signalling from the plasma membrane, the lack of CD11b significantly affected LPSinduced TLR4 endocytosis only in BM-DCs, but not in BM-MФs (Fig. 4a and Supplementary Fig. S4b). The delayed internalization was specific as Fc $\gamma$ R1 expression was largely unaffected by LPS stimulation (Fig. 4a). Consistent with the hypothesis that CD11b is part of the LPS receptor complex regulating TLR4 entry into BM-DCs, upon LPS stimulation CD11b was internalized in W/T BM-DCs, but not in BM-MФs (Fig. 4b). Likewise, LPS-induced 
a
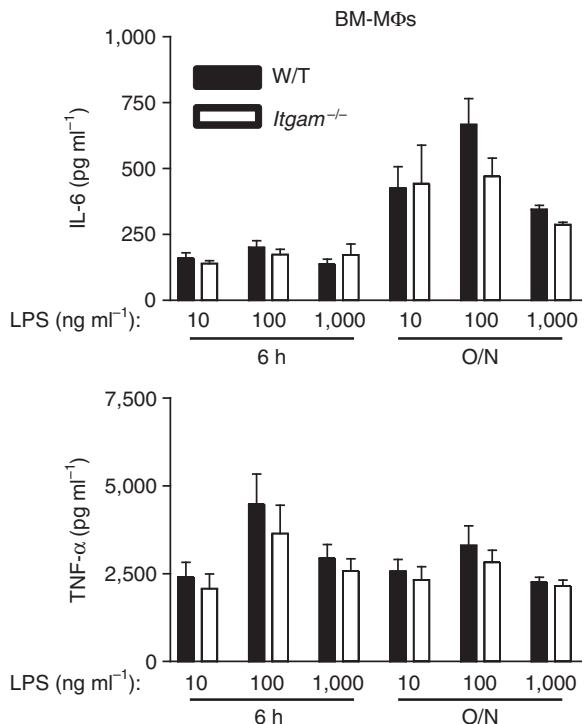

b

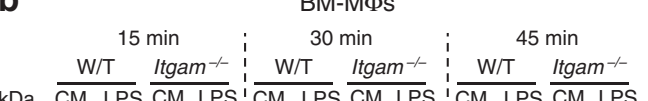

kDa CM LPS CM LPS $\overline{C M ~ L P S ~ C M ~ L P S ~} \overline{C M ~ L P S ~ C M ~ L P S ~}$
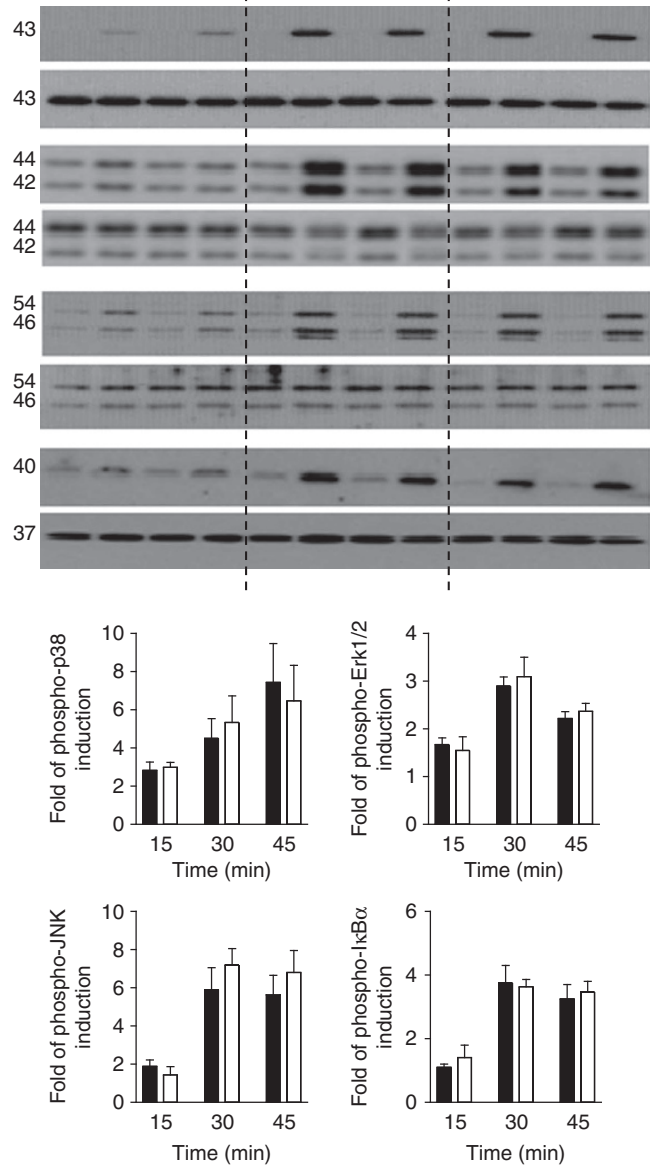

p-p38

Total p38

p-Erk1/2

Total Erk1/2

p-JNK

Total JNK

$\mathrm{p}-\mathrm{I} \kappa \mathrm{B} \alpha$

GAPDH
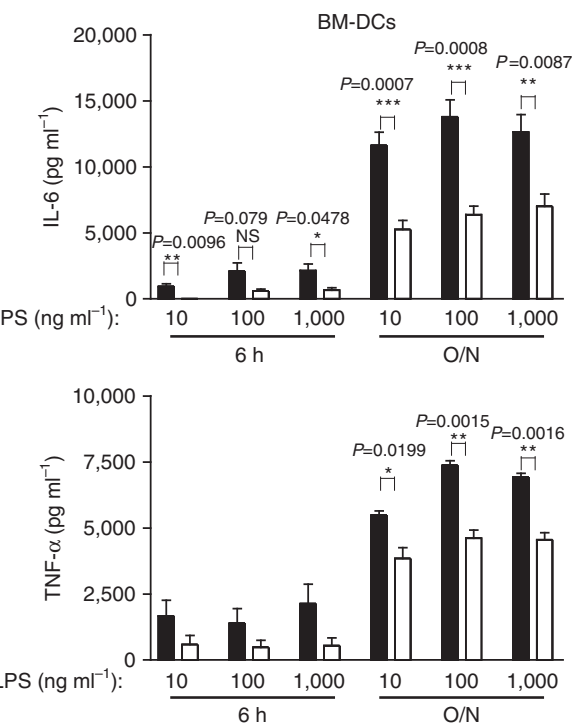

BM-DCs
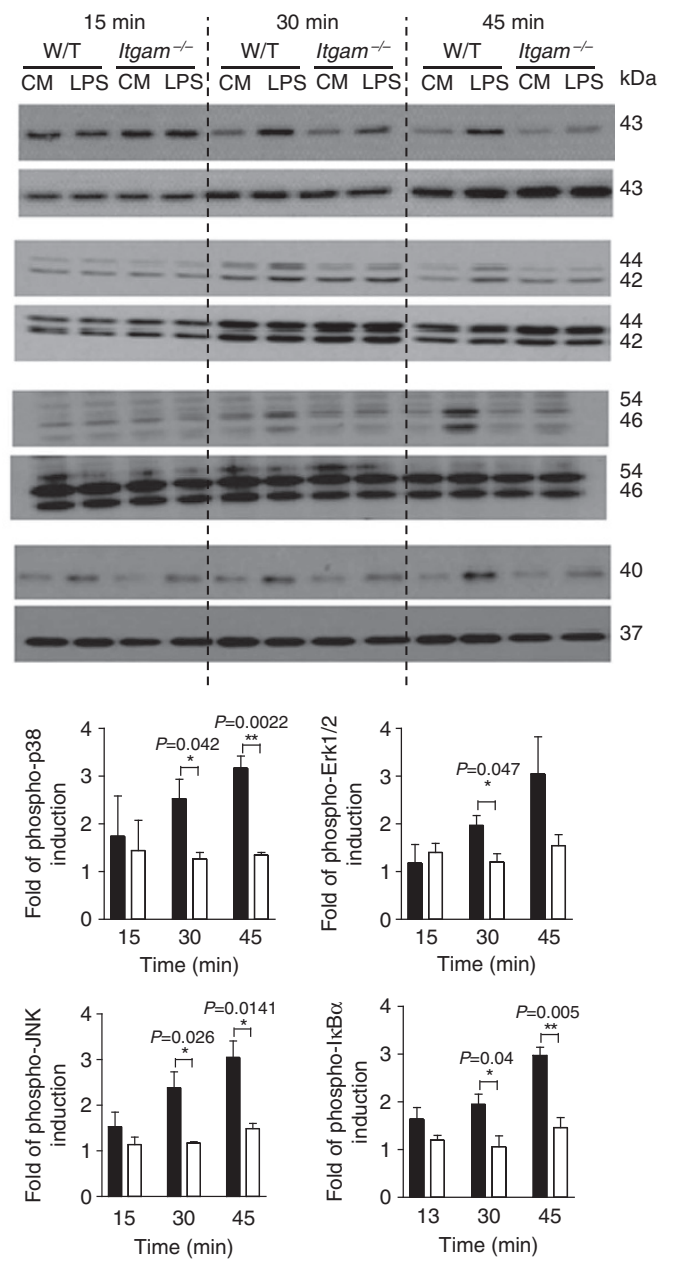

Figure 2 | CD11b is required for MyD88-dependent TLR4 signalling and LPS-induced pro-inflammatory production in DCs. (a) BM-MФs and BM-DCs from W/T or Itgam ${ }^{-1-}$ mice were treated with the doses of smooth LPS as indicated, and the production of IL- 6 and TNF- $\alpha$ was measured at $6 \mathrm{~h}$ and overnight $(\mathrm{O} / \mathrm{N})$. Data are mean \pm s.e.m. from three independent experiments, three mice per group. (b) W/T or Itgam ${ }^{-/-}$BM-MФs and BM-DCs were left untreated (CM) or treated with $1 \mu \mathrm{g} \mathrm{ml}{ }^{-1}$ of LPS for the time indicated. Phosphorylation of p38, Erk1/2, JNK and IкB $\alpha$ was examined using western blot. Quantification of band intensity showing the fold increase in phospho-protein levels relative to untreated cells and normalized to total protein levels. Data are mean \pm s.e.m. from three independent experiments. ${ }^{\star} P<0.05,{ }^{\star \star} P<0.01,{ }^{\star \star \star} P<0.005$ (t $t$-test). 

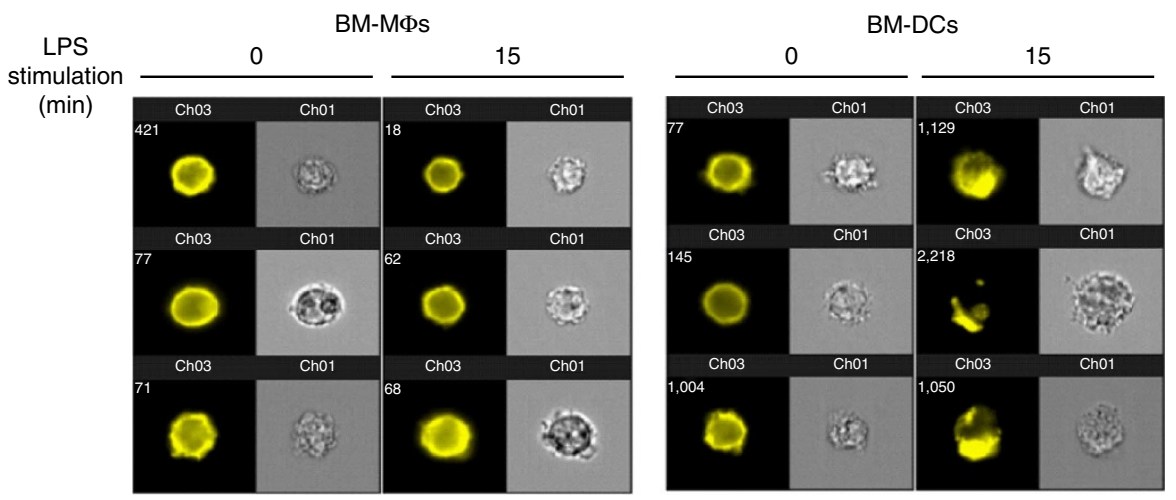

$\%$ Of increase in the cells with clustered surface CD11b

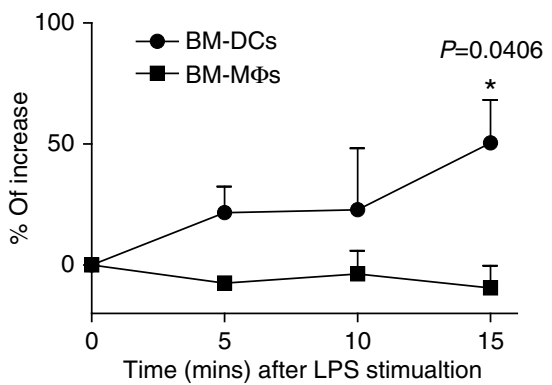

Figure 3 | LPS stimulation induces surface CD11b clustering in DCs but not in $\mathbf{M \Phi s . ~ W / T ~ B M - M \Phi s ~ a n d ~ B M - D C s ~ w e r e ~ s t i m u l a t e d ~ w i t h ~} 1 \mu \mathrm{g}^{-1} \mathrm{ml}^{-1}$ LPS for the times indicated. The percentage increase in the cells showing CD11b clustering was quantified using the ImageStream system software as described in the Methods section. Data represent mean \pm s.e.m. from three independent experiments. ${ }^{\star} P<0.05$ ( $t$-test).

CD14 internalization was not affected by the lack of CD11b in BM-MФs, but was inhibited in Itgam ${ }^{-}-$BM-DCs, indicating that the LPS-induced endocytic pathway in BM-DCs requires both molecules (Supplementary Fig. S5a). This was further investigated using confocal microscopy that showed co-staining of CD11b and CD14 in BM-DCs only after LPS stimulation (Fig. 4c). Both molecules appeared around LPS-induced macropinosomes, which stained positive for the early endosomal marker EEA1, confirming the flow cytometry data. In contrast, the expression level of CD11c (integrin $\alpha_{X}$ ) remained unchanged after LPS stimulation, demonstrating that this molecule is not involved in TLR4 endocytosis (Supplementary Fig. S5b). To demonstrate that CD11b was internalized by endocytosis like TLR4, we used dynasore that blocks endocytosis ${ }^{7}$. Dynasore treatment prevented the internalization of both TLR4 and CD11b in BM-DCs (Fig. 4d), confirming that CD11b is part of the TLR4-endocytic cluster.

CD11b is required for LPS-induced RANTES secretion by DCs. In agreement with the endocytic findings, Itgam ${ }^{-/}$BM-DCs, but not BM-MФs, were markedly defective in LPS-induced TRIFmediated RANTES production (Fig. 5a). Similar results were observed with non-adherent GM-CSF-differentiated BM-derived myeloid cells, which are widely used as BM-DCs (Supplementary Fig. S2b). Consistent with the cytokine data, TRIF-mediated TANK-binding kinase-I (TBK-1) and IRF3 phosphorylation were markedly diminished in LPS-treated Itgam $^{-1-}$ BM-DCs compared with W/T BM-DCs, but similar in BM-MФs (Fig. 5b and Supplementary Fig. S9). By contrast, no obvious differences were found in Akt or Syk phosphorylation in Itgam $^{-1-}$ BM-DCs (Supplementary Fig. S5c and S11). Stimulation of BM-DCs with LPS induces upregulation of MHC class II and co-stimulatory molecules in a TRIF-dependent manner ${ }^{29}$. In keeping with the signalling data, Itgam ${ }^{-1-}$ BM-DCs displayed markedly diminished induction of MHCII, CD80 and CD86 after LPS stimulation (Fig. 5c) compared with the W/T BM-DCs. In addition, this defect was specific for BM-DCs, as MHCII expression was comparable in LPS-treated W/T and Itgam $^{-1-}$ BM-MФs (Supplementary Fig. S5d). Collectively, these data demonstrate that $\mathrm{CD} 1 \mathrm{lb}$ contributes to deliver TLR4 to endosomes and is required to induce TRIF-dependent signalling in BM-DCs.

Endosomal TLR4 endocytosis requires CD14 (ref. 3). If the restricted role of CD11b in BM-DCs is determined at least in part by the low expression of CD14, then increasing CD14 surface expression should restore TLR4 endocytosis and TRIF-dependent signalling. To test this, we increased the expression of CD14 by stimulating BM-DCs with CpG DNA, a TLR9 ligand that does not induce TRIF-IRF3 signalling events ${ }^{3,30}$. Consistent with previous data ${ }^{3}, \mathrm{CpG}$ DNA treatment enhanced surface levels of CD14 compared with immature BM-DCs. Interestingly, this increase was even more pronounced in Itgam $^{-1}-$ BM-DCs (Fig. 6a). The CpG-matured Itgam ${ }^{-1-}$ BM-DCs, stimulated with LPS, internalized TLR4 similarly to CpG-matured W/T BM-DCs (Fig. 6a and Supplementary Fig. S10). However, the normalization in TLR4 endocytosis did not rectify the TRIFdependent phosphorylation of IRF3 (Fig. 6b), nor did it restore RANTES production in Itgam $^{-} /$BM-DCs to a level comparable to W/T BM-DCs (Fig. 6c). As TLR9 stimulation is known to involve MyD88/NF- $\kappa \mathrm{B}$ signalling, IL-6 production was not assessed. Although with this approach we cannot rule out secondary effects of the CpG treatment, these data confirm that LPS-induced TLR4 endocytosis and signalling are independent events $^{3}$ and suggest that CD11b on BM-DCs regulates TRIF/ IRF3-dependent signalling from endosomes.

T-cell response elicited by LPS-primed DCs depends on CD11b. TLR ligands such as LPS act as adjuvants for adaptive immune responses ${ }^{29}$ and TLR-induced pro-inflammatory cytokines, in particular IL-6 (ref. 31), regulate T-cell responses. To test whether the adjuvant effect of LPS required CD11b on DCs, we first examined whether the impaired cytokine response observed in LPS-treated Itgam ${ }^{-1}$ BM-DCs could influence T-cell polarization in vitro. After 3-day stimulation with conditioned medium (CM) from LPS-stimulated DCs in the presence of transforming growth factor (TGF)- $\beta$, significantly more $\mathrm{T}$ regulatory cell and fewer Th17 cells were detected in the cultures from Itgam $^{-1}$ - DCs compared with those from W/T DCs (Supplementary Fig. S6a).

We then used the male-specific Y-chromosome-encoded minor histocompatibility antigens $(\mathrm{HY})^{32,33}$ model to assess the impact in vivo. Intranasal (i.n.) administration of the MHC class IIrestricted $\mathrm{HY}$ peptide $\left(\mathrm{HY}^{\mathrm{Ab}} \mathrm{D} b y\right)$ to female mice can induce tolerance to HY-disparate skin grafts. However, this DCmediated tolerance induction can be converted to immunization if LPS is co-administrated with the peptide ${ }^{32}$. We therefore treated $\mathrm{W} / \mathrm{T}$ and Itgam $^{-1-}$ female mice with $\mathrm{HY}$ peptide alone or HY peptide plus LPS (HY peptide/LPS) and isolated splenic DCs $24 \mathrm{~h}$ after the i.n. administration. In keeping with findings using BM-DCs, we found less IL-6 and RANTES producing splenic DCs in (HY peptide/LPS)-treated Itgam $^{-1-}$ mice compared with (HY peptide/LPS)-treated controls and this 
a

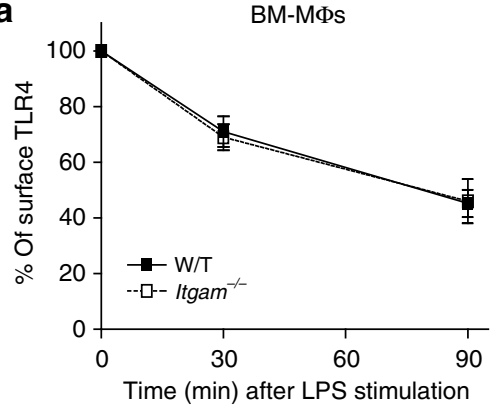

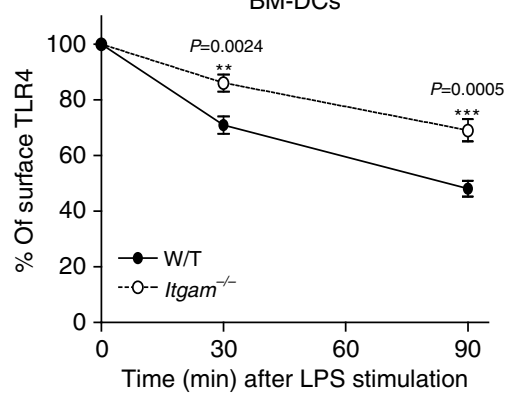

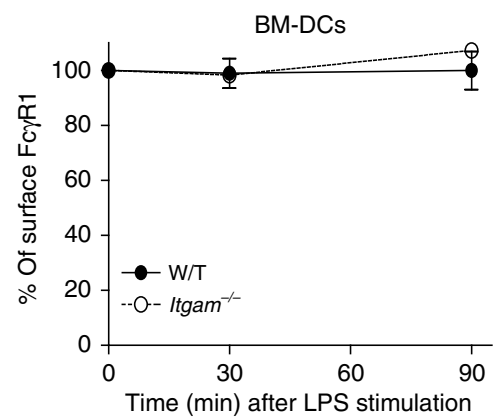

b

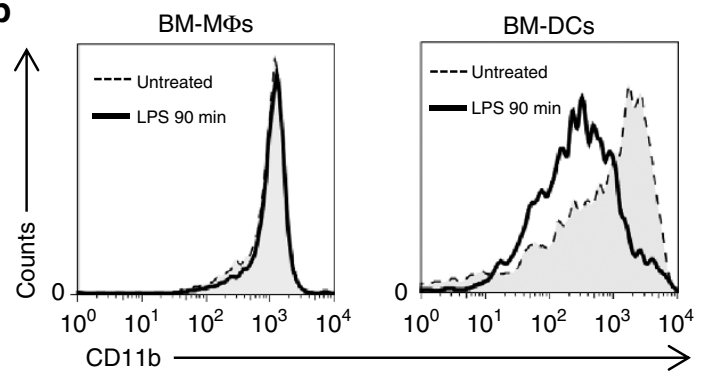

Surface CD11b

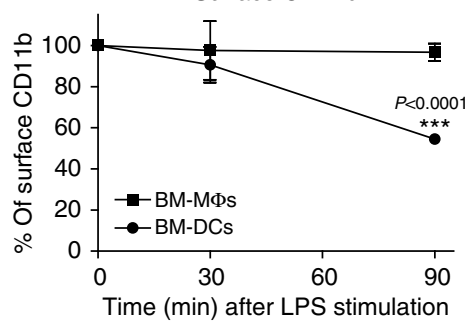

Surface TLR4

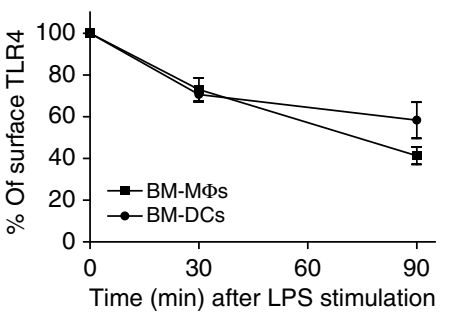

C
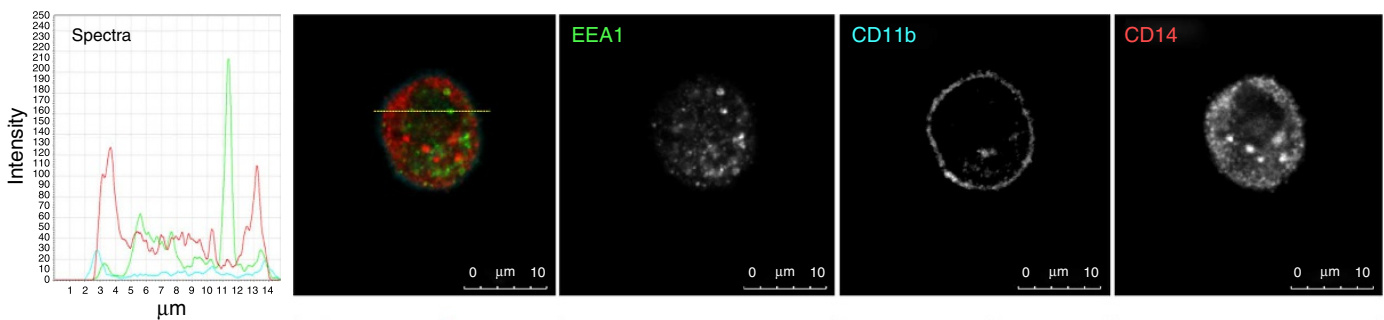

Untreated
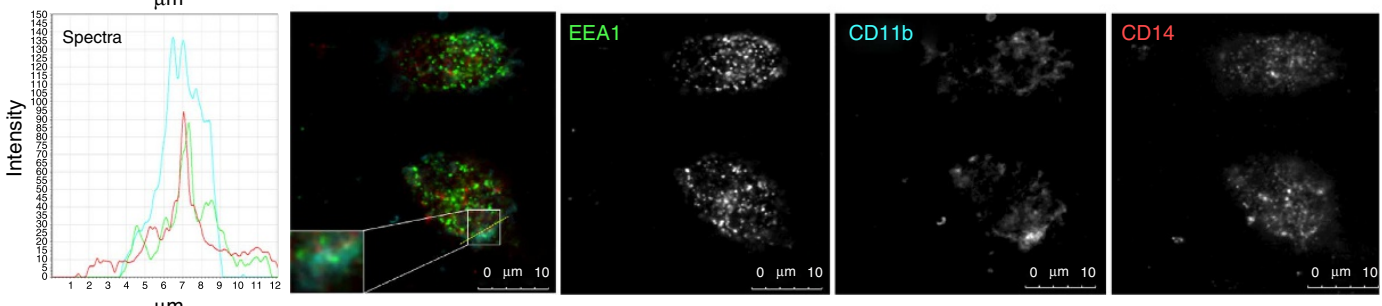

Distance $(\mu \mathrm{m})$

d

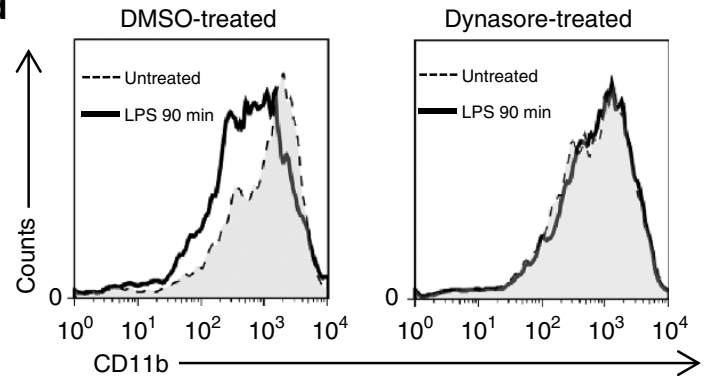

Surface CD11b

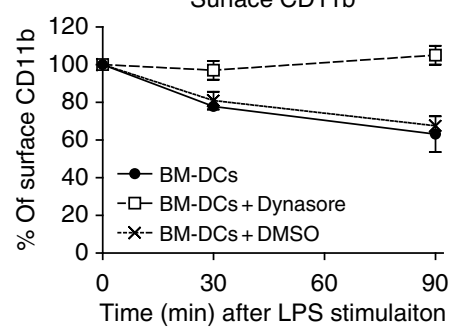

Surface TLR4

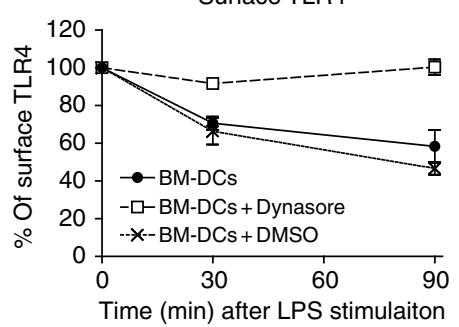

Figure 4 | LPS induces CD11b endocytosis in DCs. (a) W/T or Itgam ${ }^{-/-}$BM-MФs and BM-DCs were treated with LPS $\left(1 \mu \mathrm{g} \mathrm{ml}{ }^{-1}\right)$ for the times indicated. Receptor endocytosis was monitored with flow cytometry using the Sa15-21 mAb. Displayed are the MFIs of specific receptor staining (TLR4 or Fc $\gamma$ R1) at each time point after LPS stimulation (MFI at time 0 as 100\%). Data are representative of three independent experiments (mean $\pm \mathrm{s}$.e.m., $n=3$ ). (b) W/T BM-MФs and BM-DCs were treated with LPS $\left(1 \mu \mathrm{g} \mathrm{ml}{ }^{-1}\right)$, and surface levels of TLR4 and CD11b were assessed using flow cytometry at the times indicated. Shown are the MFIs of specific receptor staining at each time point (MFI at time 0 as 100\%). Data are representative of three independent experiments (mean \pm s.e.m., $n=3$ ). (c) W/T BM-DCs were left untreated (upper panel) or were treated with $1 \mu \mathrm{gl} \mathrm{ml}^{-1}$ of LPS (lower panel) for $30 \mathrm{~min}$. Cells were fixed, permeabilized and labelled with EEA1-alexa488 (green), CD11b-V450 (cyan) and CD14-alexa555 (red) antibodies, and analysed using confocal microscopy. Images are representative of at least two independent experiments. Scale bars $=10 \mu \mathrm{m}$. Left of the merged image plotted the florescence spectra of each channel in the region of interest (ROI; yellow line). (d) W/T BM-DCs left untreated (circle) or treated with Dynasore (square) or dimethylsulphoxide (cross) were stimulated with LPS $\left(1 \mu \mathrm{g} \mathrm{ml}{ }^{-1}\right)$. Surface levels of TLR4 and CD11b were measured at the times indicated. Displayed are the MFIs of specific receptor staining at each time point (MFI at time 0 as $100 \%)$. Data are representative of three independent experiments (mean \pm s.e.m., $n=3$ ). ${ }^{\star} P<0.05,{ }^{\star \star} P<0.01,{ }^{\star \star \star} P<0.005$ (t-test). 
a

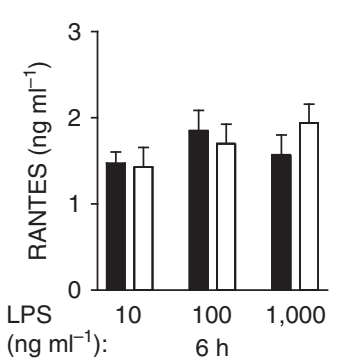

BM-MФS

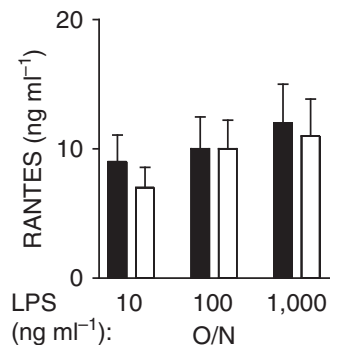

BM-DCs

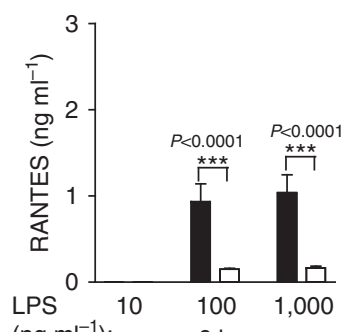

(ng ml $\mathrm{m}^{-1}$ ): $\quad 6 \mathrm{~h}$

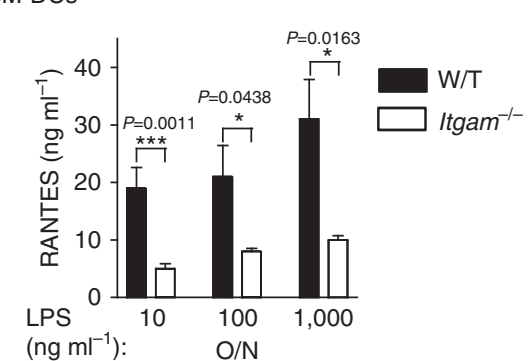

b
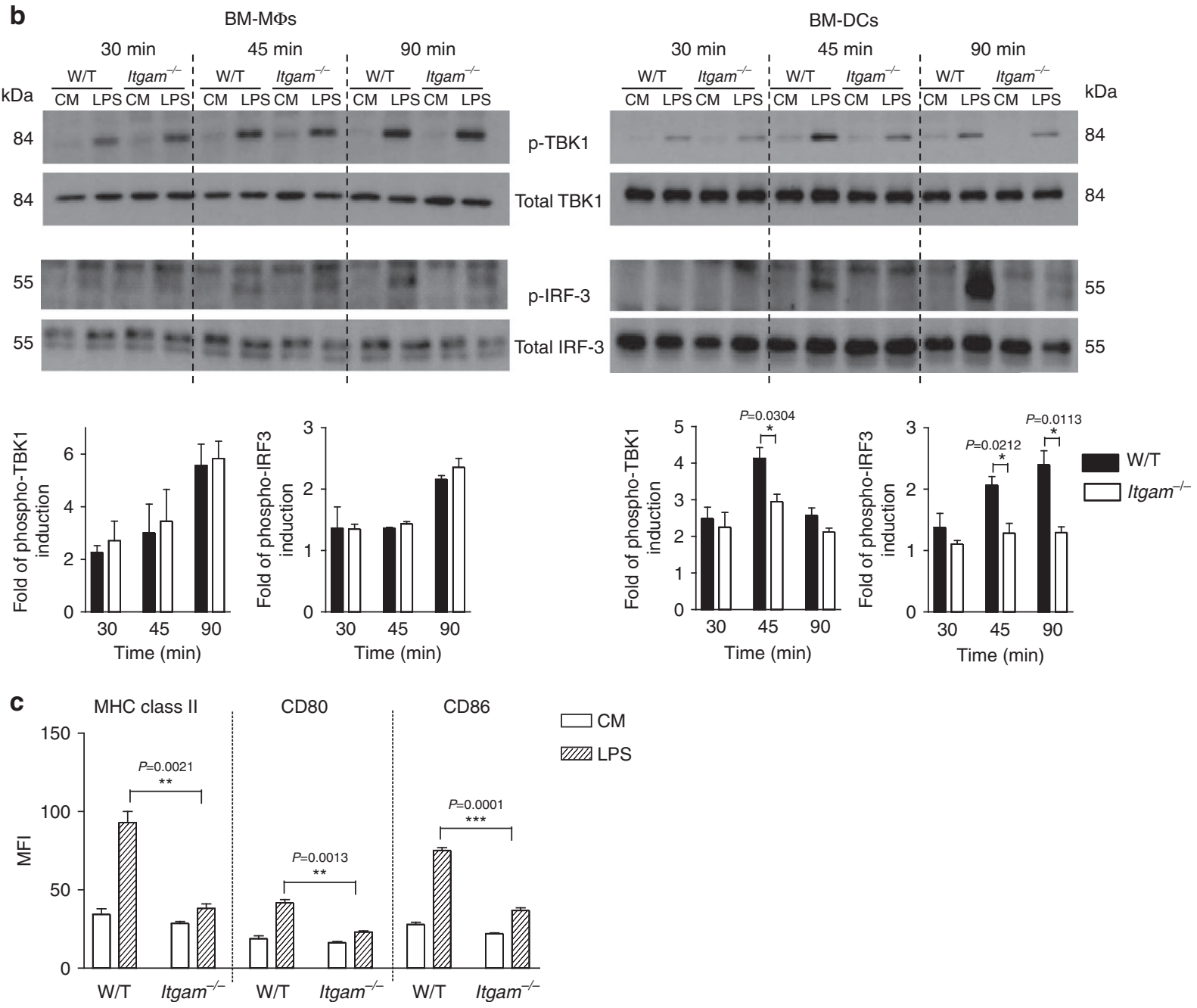

Figure 5 | CD11b is required for TRIF-dependent TLR4 signalling in DCs. (a) W/T or Itgam ${ }^{-/-}$BM-MФs and BM-DCs were treated with the indicated doses of LPS and the amounts of RANTES secreted were measured using ELISA at $6 \mathrm{~h}$ and O/N. Data are mean \pm s.e.m. from three independent experiments with three mice per group. (b) W/T or Itgam ${ }^{-/-}$BM-MФs and BM-DCs were left untreated (CM) or treated with LPS (1 $\mu$ g $\mathrm{ml}^{-1}$ ) for the time indicated. The presence of phosphorylated ( $p-$ )TBK-1 and IRF3 was assessed using western blot. Quantification of band intensity showing the fold increase in phospho-protein levels relative to untreated cells and normalized to total protein levels. Data are mean \pm s.e.m. from three independent experiments. (c) W/T or Itgam ${ }^{-/-}$BM-DCs were left untreated (CM) or treated with $1 \mu \mathrm{g} \mathrm{ml}^{-1}$ of LPS for $24 \mathrm{~h}$. DC maturation markers (MHC class II, CD80 and CD86) were assessed using flow cytometry. Data are expressed as delta MFI (MFI=test-isotype control). Data are representative of three independent experiments (mean \pm s.e.m., $n=3$ ) ${ }^{\star} P<0.05$, ${ }^{\star \star} P<0.01,{ }^{\star \star \star} P<0.005$ (t-test).

difference was not observed in the groups receiving HY peptide alone (Fig. 7a). We then assessed the function of splenic DCs by incubating them with naive $\mathrm{CD} 4^{+} \mathrm{T}$ cells isolated from HY-TCR transgenic mice (Marilyn T cells) ${ }^{34}$. As previously published ${ }^{33}$, DCs isolated from peptide-treated W/T mice induced only a very weak proliferative response of the Marilyn T cells, while the i.n. inoculation of the peptide with LPS boosted the ability of DCs to induce T-cell proliferation (Fig. 7b). Itgam ${ }^{-1-}$ splenic DCs from the (HY peptide/LPS)-treated group, however, were severely impaired in their ability to elicit proliferation of antigen-specific $\mathrm{T}$ cells (Fig. $7 \mathrm{~b}$ and Supplementary Fig. S6b). In addition, the percentage of IL- $6^{+}$DCs was reduced in the cocultures with in vivo (HY peptide/LPS)-primed Itgam $^{-1-}$ DCs compared with the W/T counterparts (Fig. 7c). By contrast, no differences were detected between W/T and Itgam ${ }^{-1}$ - DCs that had been primed with the peptide only (Fig. 7c). 
a

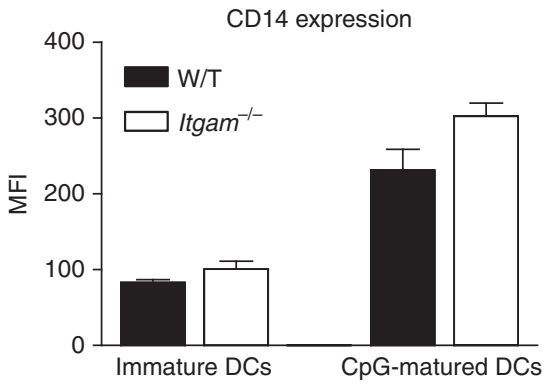

Immature BM-DCs

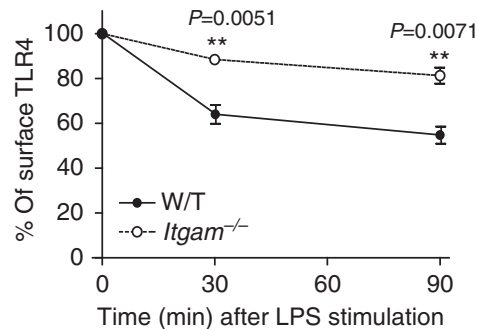

CpG-matured BM-DCs

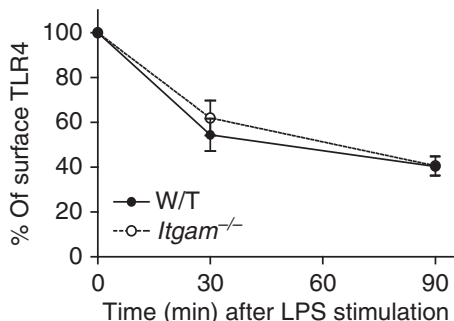

b

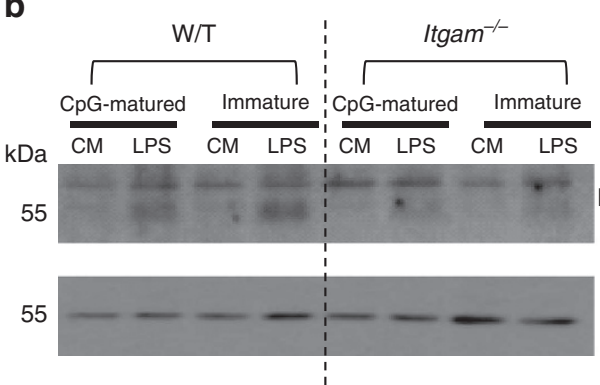

(45 $\mathrm{min})$

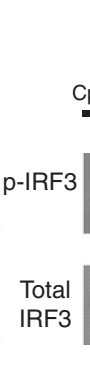

Total
IRF3

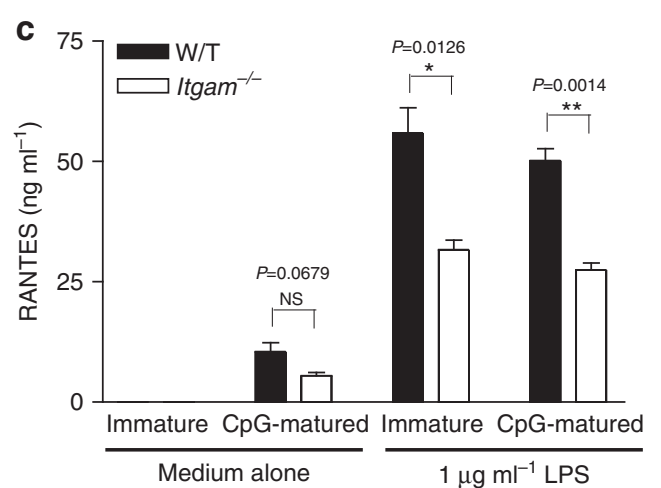

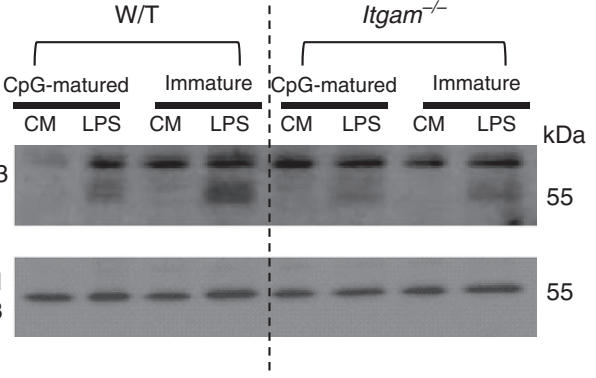

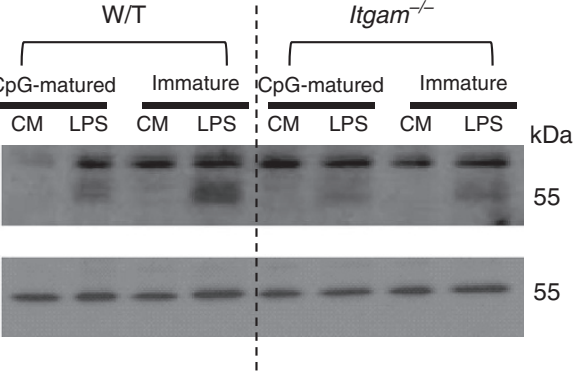

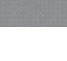

(90 $\mathrm{min})$

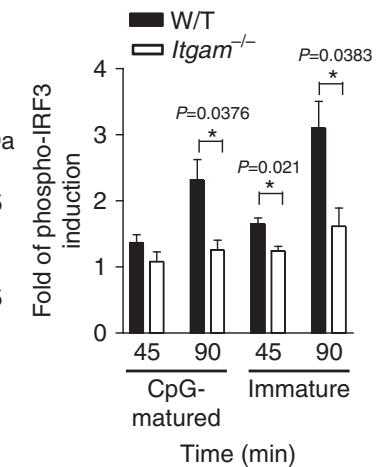

Time (min)

Figure 6 | Upregulation of CD14 expression in Itgam ${ }^{-/-}$BM-DCs matured with CpG DNA rectifies TLR4 endocytosis but not TRIF-dependent signalling events. BM-DCs were treated with $0.5 \mu \mathrm{M} \mathrm{CpG} \mathrm{DNA} \mathrm{(CpG-matured)} \mathrm{or} \mathrm{left} \mathrm{untreated} \mathrm{(immature)} \mathrm{for} 18 \mathrm{~h}$. (a) Surface expression of CD14 was assessed. Cells were then stimulated with $1 \mu \mathrm{g} \mathrm{ml} \mathrm{m}^{-1}$ of LPS and the surface levels of TLR4 were measured using flow cytometry with the Sa15-21 mAb at the times indicated. Displayed are the MFI of specific receptor staining at each time point (MFI at time 0 as $100 \%$ ). (b) Immunoblot analysis of phosphorylated ( $p$-)IRF3 in cell extracts from CpG-matured and -immatured DCs after stimulation with $1 \mu \mathrm{gl} \mathrm{ml}^{-1}$ of LPS for 45 and 90 min. Quantification of band intensity showing the fold increase in p-IRF3 levels relative to untreated cells and normalized to total IRF3 levels. Data are mean \pm s.e.m. from three independent experiments. (c) The levels of RANTES secreted were measured using ELISA $24 \mathrm{~h}$ after LPS stimulation $\left(1 \mu \mathrm{g} \mathrm{ml}{ }^{-1}\right)$. Data are representative of three independent experiments (mean \pm s.e.m., $n=3$ ). n.s $=$ nonsignificant, ${ }^{\star} P<0.05$, ${ }^{\star \star} P<0.01$ ( $t$-test).

To test the impact of the impaired TLR4 signalling in Itgam $^{-1-}$ BM-DCs in vivo, we investigated whether the absence of CD11b influenced the rejection pattern of syngeneic male skin graft on female recipients receiving the HY peptide plus LPS. First, we demonstrated that male skin grafted on untreated Itgam $^{-1-}$ females was not rejected faster than on W/T females (Fig. 7d) and that i.n. administration of $\mathrm{HY}$ peptide prolonged equally the survival of syngeneic male skin grafts (Fig. 7d). We then assessed the male graft survival after i.n. administration of HY peptide/LPS. As expected, the rejection of the male skin graft in HY peptide/LPS-pretreated W/T female mice followed a faster time course (mean survival time (MST): 15 days) than that of male skin grafts in untreated W/T females (MST: 34 days). More importantly, HY peptide/LPS-pretreated W/T female mice rejected the male skin grafts significantly faster than the Itgam $^{-1-}$ female mice with a MST of 15 and 27 days, respectively (Fig. 7e). In addition, after re-exposure to male antigen, a reduced expansion of $\mathrm{Uty}^{+} \mathrm{CD} 8{ }^{+} \mathrm{T}$ cells and $\mathrm{CD} 4{ }^{+} \mathrm{CD} 25^{+}$Foxp3 ${ }^{-}$ $\mathrm{T}$ cells was detected in HY peptide/LPS-pretreated Itgam $^{-1}-$ mice compared with their W/T counterparts, suggesting poor T-cell priming (Supplementary Fig. S6c). Taken together, these data confirmed in vivo the key modulating function of CD11b on LPSprimed DCs.

\section{Discussion}

The recognition of microbial products by TLRs can trigger innate immune responses and prime adaptive immunity. Key to a fine-tuned response is the existence of distinct responses

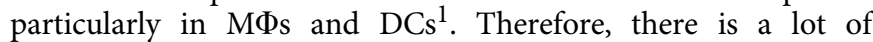
interest in understanding how TLR activation is regulated in a cell-type-specific manner. Our discovery that CD11b controls LPS-induced signalling pathways in DCs but not in MФs reconciles some of the conflicting reports in the literature and 
a
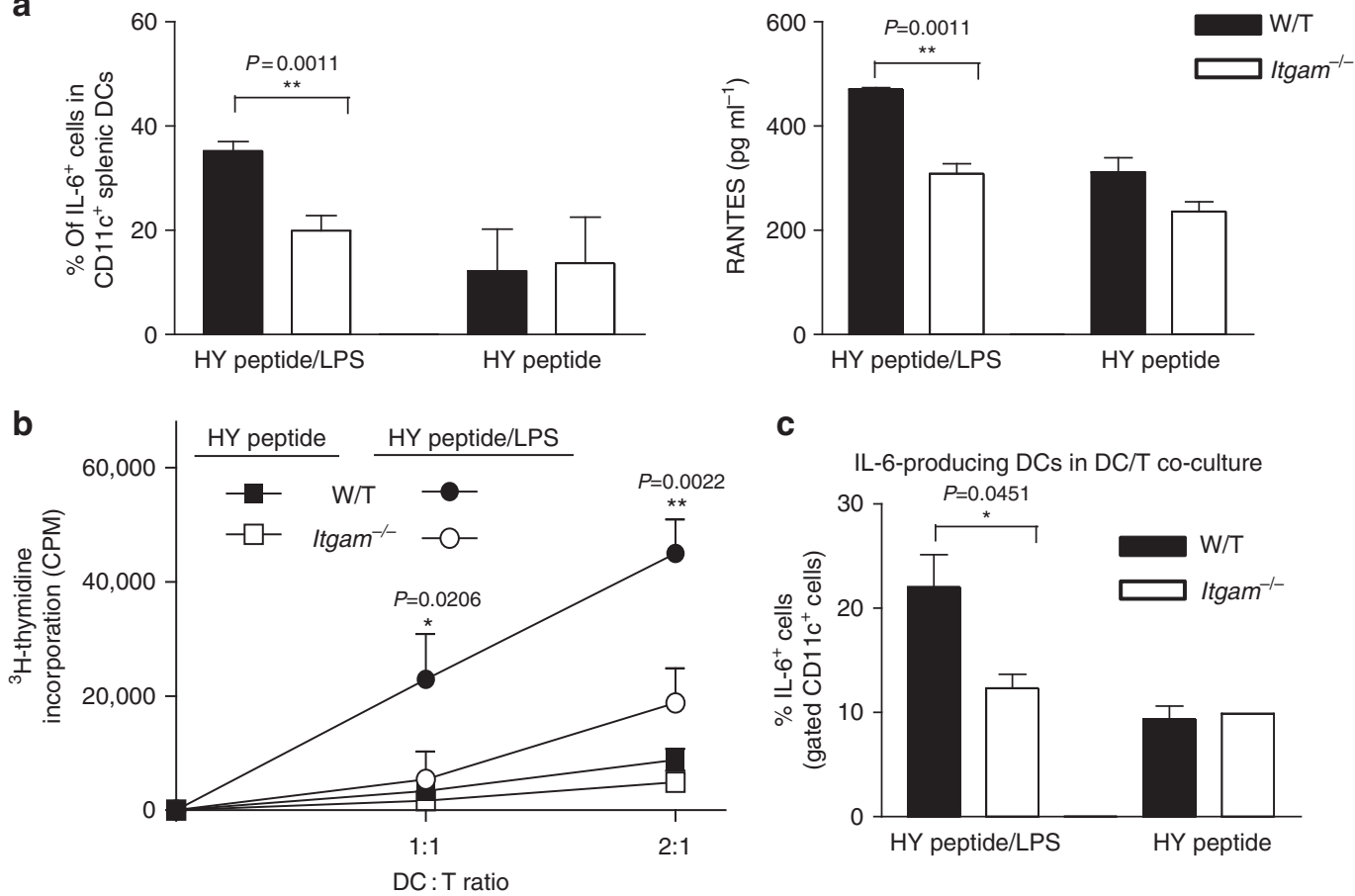

C

d
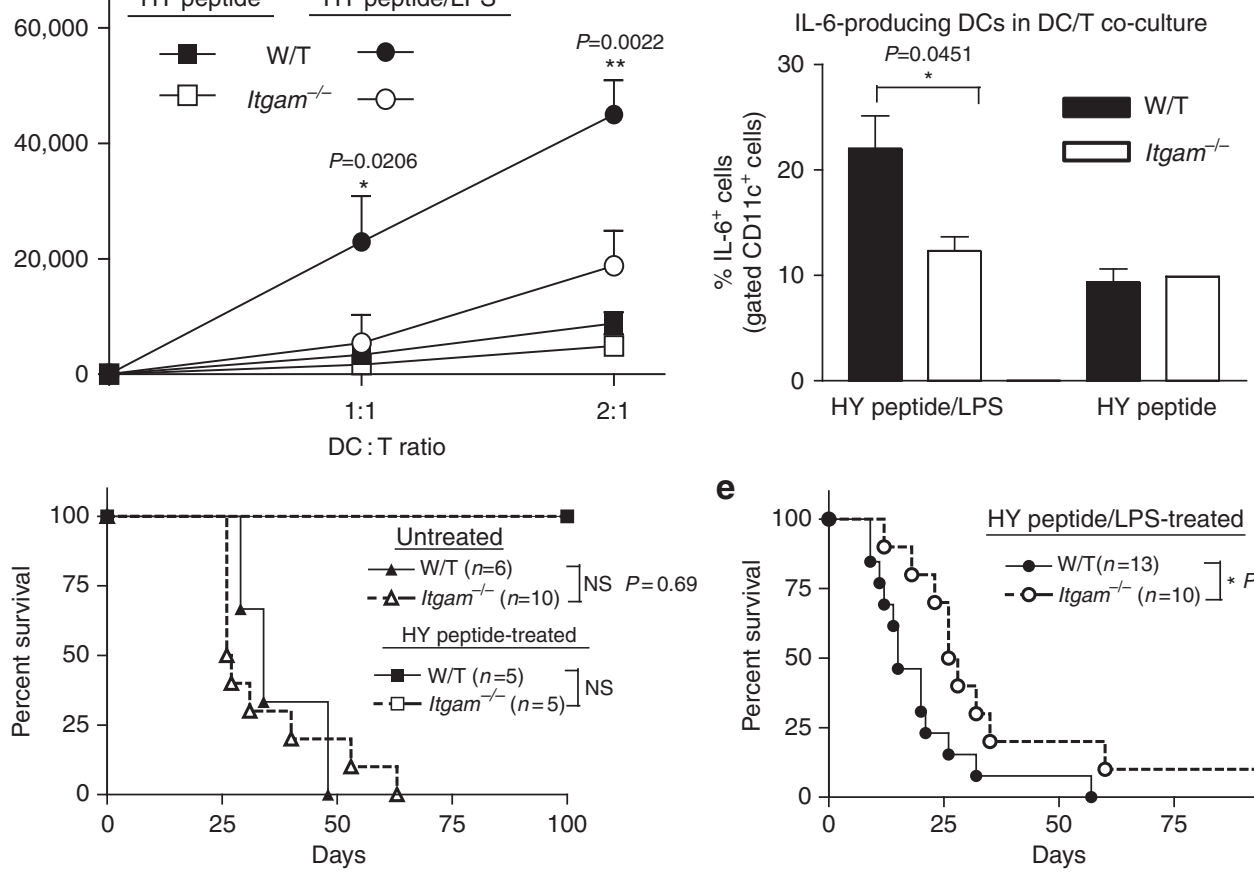

e

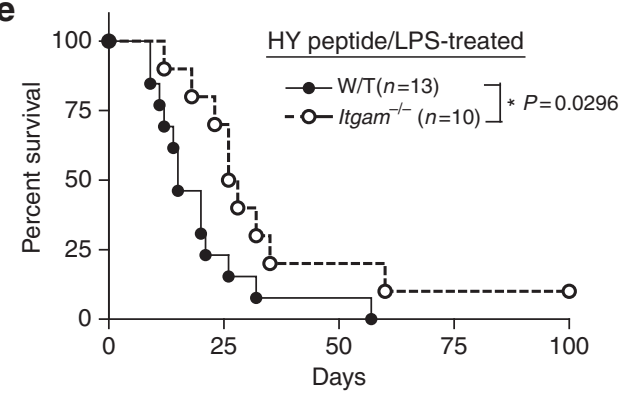

Figure 7 | CD11b is required for the LPS adjuvant effect in DCs. (a-c) Female W/T or Itgam ${ }^{-/-}$mice $(n=3)$ were given i.n. twice $100 \mu \mathrm{H}$ HYDby peptide or peptide plus $3 \mu \mathrm{g}$ LPS. Splenic CD11c ${ }^{+}$cells isolated $24 \mathrm{~h}$ later were: (a) immediately stimulated with PMA/ionomycin for $6 \mathrm{~h}$ and the $\%$ of IL- $6^{+}$cells was determined using flow cytometry. The amount of secreted RANTES at $24 \mathrm{~h}$ was measured using ELISA; (b) cocultured with Marilyn T cells and the T-cell proliferation was assessed by ${ }^{3} \mathrm{H}$-thymidine uptake at $48 \mathrm{~h}$. (c) Percentage of CD11c ${ }^{+} \mathrm{IL}-6$-producing cells in the 3 -day DC/T cocultures was quantified by intracellular staining. Data are shown as mean \pm s.e.m., $n=3,{ }^{\star} P<0.05,{ }^{\star \star} P<0.01$ ( $t$-test). (d) Survival of syngeneic male skin grafts transplanted on female W/T or Itgam ${ }^{-/-}$mice that have been treated i.n. with $\mathrm{HY}^{\mathrm{Db}}$ Uty peptide (square symbols; $n=5$ ) or left untreated (triangle symbols; $n=6-10$, log-rank test). (e) Male skin graft survival after i.n. administration of HY peptide plus $3 \mu g$ LPS. Data shown are pooled results from two independent experiments, ${ }^{\star} P<0.05$ (log-rank test).

provides the molecular basis for distinct cellular responses to microbial products.

$\mathrm{CD} 11 \mathrm{~b}$ is usually viewed as a $\mathrm{M} \Phi$-specific marker but it is highly expressed on myeloid DCs. Here we demonstrate that CD11b is a key mediator of the adjuvant effect of LPS in DCs, but not in MФs. In the absence of CD11b, DCs showed defective LPS binding to TLR4, impaired TLR4 signalling and poor induction of $\mathrm{T}$-cell responses. LPS stimulation induces CD11b clustering only in DCs that express lower levels of TLR4 and CD14 compared with $\mathrm{M} \Phi$ s and thus require additional accessory molecules to act as co-receptors for the full TLR4 signalling transduction ${ }^{16,18,35,36}$. Our study demonstrates that CD11b promotes LPS binding to TLR4 and is involved in both TLR4 signalling pathways and that the CD11b dependency is not the result of a GM-CSF-mediated cell-programming effect. In this context, it is of note that CD11b-deficient GM-CSF bone marrow-derived adherent cells (Supplementary Fig. S2), which have a MФ and DC overlapping gene expression profile ${ }^{23}$, secreted less IL-6 (MyD88-dependent) but similar amounts of RANTES (TRIF-dependent) compared with the W/T cells. The responses displayed by these cells are consistent with the findings reported by Kagan et al. ${ }^{4}$ They demonstrated that $\mathrm{CD} 11 \mathrm{~b}$ promoted TIRAP enrichment at the plasma membrane and had a positive regulatory effect restricted to the MyD88-dependent pathway ${ }^{4}$. On the basis of our observations, we would argue that these CD11b-mediated effects are likely to be restricted to a subtype of in vitro differentiated myeloid cells or a stage of the M $\Phi / D C$ lineage differentiation process.

Another novel finding of our study is the involvement of CD11b in the DC endosomal TRIF-dependent pathway. This pathway requires two steps: the initial TLR4 endocytosis and the subsequent TRIF-mediated signalling in the endosomes ${ }^{7}$. CD11b contributed to both stages. While CD14 has been shown to be essential for the delivery of TLR4 to the endosomal signalling machinery in DCs and $M \Phi s^{3}$, we found that the lack of CD11b delayed TLR4 internalization only in DCs. Consistent with this accessory role of CD11b, LPS-induced $\mathrm{Syk}^{3}$ and $\mathrm{Akt}^{37}$ activations were not affected. Notably, while enhancing CD14 surface 
expression with CpG DNA treatment could compensate the defect in TLR4 internalization, it was unable to compensate defects in the TRIF/IRF3 signalling pathway. These data suggest two separate roles of CD11b in DCs: one in promoting TLR4 endocytosis and one in TRIF-mediated signalling, the latter appears to require $\mathrm{CD} 11 \mathrm{~b}$ irrespectively of the trafficking events. Our data also highlight the importance of CD11b in DCmediated TLR4 signalling in vivo, a finding that challenges the traditional view of this molecule as a primary 'phagocytic receptor'. The impaired T-cell response induced by Itgam $^{-1-}$ DCs upon LPS stimulation is consistent with the poor priming and milder experimental allergic encephalomyelitis developed by Itgam $^{-/-}$mice when immunized with myelin oligodendrocyte glycoprotein peptide plus complete Freund's adjuvant that activates TLR4 (ref. 38). The adjuvant effect of CD11b on LPSprimed DCs provides a protective mechanism by which mature DCs retain the ability to sense and respond to microbial encounters by inducing T-cell differentiation.

The demonstration that $\mathrm{CD} 11 \mathrm{~b}$ influences the trafficking and signalling functions of TLR4 in BM-DCs but not in MФs may also explain some of the discrepancies in the literature. A celltype-specific role can reconcile the observation that CD11bdeficient cells have an impaired IL-6 production in response to LPS $^{4}$ with the finding that a tail-less version of CD11b is unable to promote phagocytosis but is still capable of eliciting a NF- $\mathrm{kB}-$ mediated response to LPS ${ }^{16}$. Similarly, the explanation for the conflicting findings that have been attributed to $\mathrm{CD} 11 \mathrm{~b}$ in various disease models ${ }^{15,38-40}$ may lie in the predominant role played by the different innate or adaptive immune cells in these experimental conditions. Furthermore, a recent study suggested that $\mathrm{CD} 11 \mathrm{~b}$, in the absence of an exogenous ligand, can serve as negative regulator of TLR-triggered inflammatory responses ${ }^{15}$. As it appears that most of the observations in this study were obtained using thioglycolate-elicited peritoneal $\mathrm{M} \Phi$ s, we would argue that the reported hyper-responsiveness of Itgam $^{-1-}$ MФs to TLR stimulation ${ }^{15}$ is restricted to thioglycolate-elicited peritoneal MФs. These MФs from Itgam ${ }^{-1-}$ mice display an abnormal state of activation prior to TLR4 stimulation that is consistent with previous observations ${ }^{21,22}$, and the knowledge that thioglycolate-induced peritonitis requires complement activation $^{20}$. Our data, however, do not exclude the possibility that ligation of $\mathrm{CD} 11 \mathrm{~b}$ to fibrinogen or $\mathrm{iC} 3 \mathrm{~b}$ can modulate TLR4 signalling in MФs. Wang et al. ${ }^{12}$ reported indirect inhibitor effects mediated by activated CR3 when ligated with fibrinogen and we have obtained similar results using iC3b-coated particles (Supplementary Fig. S7). Interestingly, we found that this negative effect on TLR-triggered inflammatory responses is restricted to MФs (Supplementary Fig. S7), suggesting a dynamic cross-talk between TLRs and integrins whose outcome is, at least in part, dependent on cell lineage/differentiation. Collectively, our data and previous reports support the hypothesis of a 'signal-switch' model ${ }^{41}$ whereby at sites of an acute inflammation a strong CR3-mediated inhibitory signalling effect on $\mathrm{M} \Phi$ s may contribute to dampen the potential toxicity of the cytokines generated by TLR stimulation, while during chronic inflammation or in steady-state conditions, tonic CD11b signalling triggered by low-avidity receptor ligation has no detectable effects on TLR responses by MФs. These dual roles of CD11b on MФs according to the surrounding would limit the tissue damage during an acute infection without compromising tissue homoeostasis.

In summary, our work demonstrates that CD11b facilitates LPS binding to TLR4 and controls trafficking and signalling functions of TLR4 in a cell-type-specific manner by acting as an important molecule in the cross-talk between adaptive and innate immune responses. From an evolutionary perspective, these differential effects of CD11b in MФs and DCs ensure a coordinated and fine-tuned cellular response appropriate for the environmental trigger.

\section{Methods}

Mice. C57BL/6 mice were purchased from Charles River UK. C57BL/6-CD11b ${ }^{-1-}$ mice $\left(\text { Itgam }^{-/-}\right)^{21}$ were from Jackson Laboratory (Bar Harbor). Rag2 ${ }^{-I-}$ Marilyn mice (TCR transgenic for the $\mathrm{HY}^{\mathrm{Ab}} \mathrm{Db}$ y peptide NAGFNSNRANSSRSS) were a kind gift from Dr O Lant ${ }^{34}$. Two to three months old female mice were used. All animals were handled in accordance with the institutional guidelines and procedures approved by the UK Home Office in accordance with the Animals (Scientific Procedures) Act 1986.

Cell culture. Thioglycolate-elicited MФs were isolated on day 4 from the peritoneum of mice injected intraperitoneally with $0.5 \mathrm{ml}$ of $3 \%$ thioglycolate. Resident peritonea MФs were recovered from naive mice. BM-MФs and BM-DCs were generated by culturing bone marrow cells with $20 \mathrm{ng} \mathrm{ml}^{-1} \mathrm{rM}$-CSF (PeproTech) for 7 days and with $16.67 \mathrm{ng} \mathrm{ml}^{-1} \mathrm{rGM}$-CSF (PeproTech) plus rIL-4 (5 $\mathrm{ng} \mathrm{ml}^{-1}$, PeproTech) for 6 days, respectively. Cells were stimulated with TLR4-grade LPS from Escherichia Coli O111:B4 (Alexis Biochemicals). To enhance surface CD14 expression, BM-DCs were stimulated with $0.5 \mu \mathrm{M} \mathrm{GpG}$ (ODN 1826, Alexis Biochemicals) for $18 \mathrm{~h}$. Splenic DCs were isolated by CD11c MACs beads (Miltenyi Biotec) and Marilyn cells were purified with $\mathrm{CD}^{+}{ }^{+} \mathrm{T}$ cells isolation kit II (Miltenyi Biotec).

Flow cytometry. BM-MФs and BM-DCs were stained with the following antibodies for phenotypic analysis: Fluorochrome-conjugated monoclonal antibodies against CD11b (M1/70; dilution 1:200), CD11c (N418; dilution 1:100), CD115 (AFS98; dilution 1:100), CD80 (16-10A1; dilution 1:100), CD86 (GL1; dilution 1:100), F4/80(BM8; dilution 1:50) and MHC class II (AF6-120.1; dilution 1:100) were from BD Biosciences Pharmingen. Monoclonal antibodies against TLR4 (Sa15-21 and MTS510) were from BioLegend. The Sa15-21 antibody was labelled with biotin (Sigma). Both anti-TLR4 antibodies were added at a dilution of 1:50. PerCp-Cy5.5-conjugated (dilution 1:50) and biotin-conjugated (dilution 1:100) monoclonal antibodies against CD14 (Sa2-8) were from eBioscience. Data were acquired using a FACSCalibur (Becton-Dickinson) and expressed as delta the mean fluorescent intensity (MFI of the sample - MFI of the isotype control).

ImageStream data acquisition and analysis. Cells were stimulated with $1 \mu \mathrm{g} \mathrm{ml}^{-1}$ of LPS in polypropylene tube for different time points. After being fixed with $1 \%$ paraformaldehyde the cells were stained with PE-conjugated anti-mouse CD11b (dilution 1:100). Images of 10,000 cells were acquired for each sample on the ImageStream imaging cytometer System 100 (Amnis Corp). The data were analysed using the ImageStream Data Exploration and Analysis Software (IDEAS, Amnis). Receptor clustering was analysed as described ${ }^{42}$.

Immunofluorescence microscopy. BM-DCs were grown on Bio-coat coverslips (BD biosciences), fixed in $4 \%$ paraformaldehyde, permeabilized with $0.1 \%$ saponin and blocked with 5\% goat serum. Samples were incubated with anti-CD11b-V450 (BD Biosciences, dilution 1:50), anti-CD14-biotin (ebioscience; dilution 1:50) and rabbit anti-EEA1 (Cell Signaling Technology; dilution 1:100). Secondary antibodies Alexa Fluor 555-conjugated Streptavidin and Alexa Fluor 488-conjugated goatanti-rabbit IgG were both from Molecular Probes and were added at a dilution of 1:100. Samples were then analysed with a Leica SP5 confocal microscope. Colocalization spectra of each channel in the region of interest were analysed using the Lecia LAS AF software.

Immunoblotting. After LPS stimulation, cells were lysed with RIPA buffer (Sigma) in the presence of protease inhibitor cocktail (Sigma) and phosphatase inhibitor cocktail 2 (Sigma). Proteins were fractioned using 10 or $12 \%$ SDS-PAGE and analysed using immunoblotting with the following antibodies: antibody specific for p38 MAPK phosphorylated at Thr180/Tyr182 (3D7), Akt phosphorylated at Ser473, Erk1/2 phosphorylated at Thr202/Tyr204 (197G2), IRF3 phosphorylated at Ser396 (4D4G), I $\mathrm{B} \alpha$ phosphorylated at Ser32/36 (5A5), JNK phosphorylated at Thr183/185 (81E11), Syk phosphorylated at Tyr525/526 (C87C1), TBK-1 phosphorylated at Ser172 (D52C2), p38 MAPK, Akt, Erk1/2 (137F5), IRF3(D83B9), JNK, Syk, TBK-1 (D1B4) and GAPDH (14C10). All antibodies were from Cell Signaling Technology and were used at a dilution of 1:1,000. Densitometry analysis on the bands was performed using the NIH Image J software and normalizing the data to total protein levels.

T-cell polarization assay. BM-DCs were stimulated with $1 \mu \mathrm{g} \mathrm{ml}^{-1} \mathrm{LPS}$, and CM was collected after $18 \mathrm{~h}$. CD4 ${ }^{+} \mathrm{T}$ cells were purified from naive W/T mice and activated with plate-bound anti-CD3 $\left(2 \mu \mathrm{g} \mathrm{ml}^{-1}\right.$; Biolegend $)$ and soluble antiCD28 (5 $\mathrm{g} \mathrm{ml}^{-1}$; eBioscience) in the presence of recombinant TGF- $\beta 1$ (5 $\mathrm{ng} \mathrm{ml}^{-1}$, PeproTech). CM from W/T or Itgam $^{-1}$ - DCs was added to the T-cell culture at a ratio of 1:1. On day 3, the cells were restimulated with $10 \mathrm{ng} \mathrm{ml}^{-1}$ of PMA (Sigma) and $250 \mathrm{ng} \mathrm{ml}^{-1}$ of Ionomycin (Calbiochem) in the presence of 
GolgiStop (BD Pharmingen) for $5 \mathrm{~h}$. Cells were then intracellularly stained with PE-conjugated anti-IL-17 (TC11-18H10; dilution 1:100) and FITC-labelled anti-IFN- $\gamma$ (XMG1.2; dilution 1:100) (BD Pharmingen). For the detection of $\mathrm{T}$ regulatory cells, cells were intracellularly stained with antigen-presenting cellconjugated Foxp3 (FJK-16s; dilution 1:100) (eBioscience) before the PMA/Ionomycin re-stimulation.

Antigen presentation assay and skin graft. Two doses of $100 \mu \mathrm{g} \mathrm{HYA}^{\mathrm{b}} D b y$ peptide with and without $3 \mu \mathrm{g}$ LPS were administered intranasally to mice. Splenic DCs were isolated 1 day after. Marilyn cells were added to DCs at 1:1 or 2:1 ratio. T-cell proliferation was assessed by thymidine incorporation after $48 \mathrm{~h}$. To assess IL-6-producing cells in the coculture, cells were restimulated with $10 \mathrm{ng} \mathrm{ml}^{-1}$ of PMA and $250 \mathrm{ng} \mathrm{ml}^{-1}$ of Ionomycin in the presence of GolgiStop for $5 \mathrm{~h}$. Phosphate-buffered saline $(20 \mu \mathrm{l})$ containing $100 \mu \mathrm{g} \mathrm{HYA} \mathrm{H}^{\mathrm{b}} \mathrm{Dby}$ peptide with or without $3 \mu \mathrm{g}$ LPS were administered intranasally on three consecutive days to $\mathrm{W} / \mathrm{T}$ and Itgam $^{-1-}$ female mice. Ten days later, the mice were grafted with syngeneic male and female skin on the lateral thorax ${ }^{32}$.

Detection of HY-specific T cells in grafted mice. One hundred days after skin grafting, mice were boosted with $5 \times 10^{6}$ male splenocytes intraperitoneally. Seven days later, spleen cells $\left(2 \times 10^{6}\right)$ were cultured with irradiated male splenocytes $\left(2 \times 10^{6}\right)$ and $10 \mathrm{IU} \mathrm{ml}^{-1}$ recombinant mouse IL-2 (PeproTech). Live cells were harvested on day 6 , cells were stained with CD8a (FITC-labelled anti-mouse CD8a, 53-6.7; dilution 1:100) and $\mathrm{HY}^{\mathrm{Db}} U$ ty PE tetramer (ProImmune; $3 \mu \mathrm{l}$ ) to detect $\mathrm{HY}$-specific $\mathrm{CD} 88^{+} \mathrm{T}$ cells. To determine the percentage of activated $\mathrm{CD} 4{ }^{+} \mathrm{T}$ cells, cells were stained with CD4 (FITC-labelled anti-mouse CD4, L3T4), CD25 (PE-labelled anti-mouse CD25, PC61) and Foxp3 (APC-labelled anti-mouse Foxp3, FJK-16s); antibodies were added at 1:100 dilution.

Statistical analysis. Comparisons between two groups were performed using two-tailed unpaired Student's $t$-test or Mann-Whitney test. Graft survival was compared using the log-rank (Mantel-Cox) test. Statistical significance is defined as $P<0.05$.

\section{References}

1. Zanoni, I. \& Granucci, F. Differences in lipopolysaccharide-induced signaling between conventional dendritic cells and macrophages. Immunobiology 215, 709-712 (2010).

2. Hashimoto, D., Miller, J. \& Merad, M. Dendritic cell and macrophage heterogeneity in vivo. Immunity 35, 323-335 (2011).

3. Zanoni, I. et al. CD14 controls the LPS-induced endocytosis of Toll-like receptor 4. Cell 147, 868-880 (2011).

4. Kagan, J. C. \& Medzhitov, R. Phosphoinositide-mediated adaptor recruitment controls Toll-like receptor signaling. Cell 125, 943-955 (2006).

5. Ostuni, R., Zanoni, I. \& Granucci, F. Deciphering the complexity of Toll-like receptor signaling. Cell Mol. Life Sci. 67, 4109-4134 (2010).

6. Fitzgerald, K. A. et al. LPS-TLR4 signaling to IRF-3/7 and NF-kappaB involves the toll adapters TRAM and TRIF. J. Exp. Med. 198, 1043-1055 (2003).

7. Kagan, J. C. et al. TRAM couples endocytosis of Toll-like receptor 4 to the induction of interferon-beta. Nat. Immunol. 9, 361-368 (2008).

8. Triantafilou, M. \& Triantafilou, K. Lipopolysaccharide recognition: CD14, TLRs and the LPS-activation cluster. Trends. Immunol. 23, 301-304 (2002).

9. Ross, G. D. Role of the lectin domain of Mac-1/CR3 (CD11b/CD18) in regulating intercellular adhesion. Immunol. Res. 25, 219-227 (2002).

10. Abram, C. L. \& Lowell, C. A. The ins and outs of leukocyte integrin signaling. Annu. Rev. Immunol. 27, 339-362 (2009).

11. Hajishengallis, G. \& Lambris, J. D. Crosstalk pathways between Toll-like receptors and the complement system. Trends. Immunol. 31, 154-163 (2010).

12. Wang, L. et al. Indirect inhibition of Toll-like receptor and type I interferon responses by ITAM-coupled receptors and integrins. Immunity 32, 518-530 (2010).

13. Fan, S. T. \& Edgington, T. S. Coupling of the adhesive receptor CD11b/CD18 to functional enhancement of effector macrophage tissue factor response. J. Clin. Invest. 87, 50-57 (1991).

14. Rezzonico, R., Imbert, V., Chicheportiche, R. \& Dayer, J. M. Ligation of CD11b and $\mathrm{CD} 11 \mathrm{c}$ beta(2) integrins by antibodies or soluble CD23 induces macrophage inflammatory protein 1alpha (MIP-1alpha) and MIP-1beta production in primary human monocytes through a pathway dependent on nuclear factor-kappaB. Blood 97, 2932-2940 (2001).

15. Han, C. et al. Integrin CD11b negatively regulates TLR-triggered inflammatory responses by activating Syk and promoting degradation of MyD88 and TRIF via Cbl-b. Nat. Immunol. 11, 734-742 (2010).

16. Ingalls, R. R., Arnaout, M. A. \& Golenbock, D. T. Outside-in signaling by lipopolysaccharide through a tailless integrin. J. Immunol. 159, 433-438 (1997).

17. Flo, T. H. et al. Involvement of CD14 and beta2-integrins in activating cells with soluble and particulate lipopolysaccharides and mannuronic acid polymers. Infect. Immun. 68, 6770-6776 (2000).
18. Scott, M. J. \& Billiar, T. R. Beta2-integrin-induced p38 MAPK activation is a key mediator in the CD14/TLR4/MD2-dependent uptake of lipopolysaccharide by hepatocytes. J. Biol. Chem. 283, 29433-29446 (2008).

19. Perera, P. Y. et al. CD11b/CD18 acts in concert with CD14 and Toll-like receptor (TLR) 4 to elicit full lipopolysaccharide and taxol-inducible gene expression. J. Immunol. 166, 574-581 (2001).

20. Taylor, P. R. et al. A hierarchical role for classical pathway complement proteins in the clearance of apoptotic cells in vivo. J. Exp. Med. 192, 359-366 (2000).

21. Coxon, A. et al. A novel role for the beta 2 integrin CD11b/CD18 in neutrophil apoptosis: a homeostatic mechanism in inflammation. Immunity 5, 653-666 (1996).

22. Cao, C., Lawrence, D. A., Strickland, D. K. \& Zhang, L. A specific role of integrin Mac-1 in accelerated macrophage efflux to the lymphatics. Blood 106, 3234-3241 (2005).

23. Lacey, D. C. et al. Defining GM-CSF- and macrophage-CSF-dependent macrophage responses by in vitro models. J. Immunol. 188, 5752-5765 (2012)

24. Krausgruber, T. et al. IRF5 promotes inflammatory macrophage polarization and TH1-TH17 responses. Nat. Immunol. 12, 231-238 (2011).

25. Inaba, K. et al. Generation of large numbers of dendritic cells from mouse bone marrow cultures supplemented with granulocyte/macrophage colonystimulating factor. J. Exp. Med. 176, 1693-1702 (1992).

26. Rosas, M. et al. The induction of inflammation by dectin-1 in vivo is dependent on myeloid cell programming and the progression of phagocytosis. J. Immunol. 181, 3549-3557 (2008)

27. Wright, S. D., Levin, S. M., Jong, M. T., Chad, Z. \& Kabbash, L. G. CR3 (CD11b/CD18) expresses one binding site for Arg-Gly-Asp-containing peptides and a second site for bacterial lipopolysaccharide. J. Exp. Med. 169, 175-183 (1989).

28. Akashi, S. et al. Lipopolysaccharide interaction with cell surface Toll-like receptor 4-MD-2: higher affinity than that with MD-2 or CD14. J. Exp. Med. 198, 1035-1042 (2003)

29. Hoebe, K. et al. Upregulation of costimulatory molecules induced by lipopolysaccharide and double-stranded RNA occurs by Trif-dependent and Trif-independent pathways. Nat. Immunol. 4, 1223-1229 (2003).

30. Blasius, A. L. \& Beutler, B. Intracellular toll-like receptors. Immunity 32, 305-315 (2010).

31. Jones, S. C., Brahmakshatriya, V., Huston, G., Dibble, J. \& Swain, S. L. TLRactivated dendritic cells enhance the response of aged naive CD4 T cells via an IL-6-dependent mechanism. J. Immunol. 185, 6783-6794 (2010).

32. Chai, J. G., James, E., Dewchand, H., Simpson, E. \& Scott, D. Transplantation tolerance induced by intranasal administration of HY peptides. Blood 103, 3951-3959 (2004).

33. Derbyshire, K. et al. Molecular mechanisms of induction of antigen-specific allograft tolerance by intranasal peptide administration. J. Immunol. 186, 5719-5728 (2011).

34. Lantz, O., Grandjean, I., Matzinger, P. \& Di Santo, J. P. Gamma chain required for naive $\mathrm{CD} 4+\mathrm{T}$ cell survival but not for antigen proliferation. Nat. Immunol. 1, 54-58 (2000).

35. Shimazu, R. et al. MD-2, a molecule that confers lipopolysaccharide responsiveness on Toll-like receptor 4. J. Exp. Med. 189, 1777-1782 (1999).

36. Triantafilou, K., Triantafilou, M. \& Dedrick, R. L. A CD14-independent LPS receptor cluster. Nat. Immunol. 2, 338-345 (2001).

37. Aksoy, E. et al. The p110delta isoform of the kinase PI(3)K controls the subcellular compartmentalization of TLR4 signaling and protects from endotoxic shock. Nat. Immunol. 13, 1045-1054 (2012).

38. Bullard, D. C. et al. Critical requirement of CD11b (Mac-1) on T cells and accessory cells for development of experimental autoimmune encephalomyelitis. J. Immunol. 175, 6327-6333 (2005).

39. Shimizu, K. et al. Leukocyte integrin Mac-1 promotes acute cardiac allograft rejection. Circulation 117, 1997-2008 (2008).

40. Ehirchiou, D. et al. CD11b facilitates the development of peripheral tolerance by suppressing Th17 differentiation. J. Exp. Med. 204, 1519-1524 (2007).

41. Ivashkiv, L. B. Cross-regulation of signaling by ITAM-associated receptors. Nat. Immunol. 10, 340-347 (2009).

42. Lapilla, M. et al. Histamine regulates autoreactive T cell activation and adhesiveness in inflamed brain microcirculation. J. Leukoc. Biol. 89, 259-267 (2011).

\section{Acknowledgements}

We thank the staff of the Biological Services Unit at our institution for the care of the animals involved in this study. This work was supported by the Wellcome Trust (grant number 088517) and in part by Cancer Research UK programme grant A15115 to G.F.

\section{Author contributions}

G.S.L. performed the experiments, interpreted the data and wrote the manuscript; J.B., K.J.W., M.S. and L.F.-J. performed the experiments; P.R.T. designed experiments 
and edited the manuscript; D.S. assisted with data interpretation; G.F. and H.T.C. discussed the data and edited the manuscript; M.B. conceived, designed the study and wrote the manuscript.

\section{Additional information}

Supplementary Information accompanies this paper at http://www.nature.com/ naturecommunications

Competing financial interests: The authors declare no competing financial interests.
Reprints and permission information is available online at http://npg.nature.com/ reprintsandpermissions/

How to cite this article: Ling, G. S. et al. Integrin CD11b positively regulates TLR4-induced signalling pathways in dendritic cells but not in macrophages. Nat. Commun. 5:3039 doi: 10.1038/ncomms4039 (2014).

(c) (i) This article is licensed under a Creative Commons Attribution 3.0 Unported Licence. To view a copy of this licence visit http:// creativecommons.org/licenses/by/3.0/. 